\title{
Chronic central serous chorioretinopathy: long- term follow-up and vision-related quality of life
}

This article was published in the following Dove Press journal:

Clinical Ophthalmology

20 December 2016

Number of times this article has been viewed

\author{
Myrte B Breukink ${ }^{1, *}$ \\ Alexander JM Dingemans ${ }^{1, *}$ \\ Anneke I den Hollander ${ }^{1,2}$ \\ Jan EE Keunen' \\ Robert E MacLaren ${ }^{3,4}$ \\ Sascha Fauser ${ }^{5}$ \\ Giuseppe Querques ${ }^{6}$ \\ Carel B Hoyng' \\ Susan M Downes 3,4 \\ Camiel JF Boon ${ }^{1,7}$ \\ 'Department of Ophthalmology, \\ ${ }^{2}$ Department of Human Genetics, \\ Radboud University Medical Center, \\ Nijmegen, the Netherlands; ${ }^{3}$ Oxford \\ Eye Hospital, Oxford University \\ Hospitals NHS Trust, ${ }^{4}$ Nuffield \\ Laboratory of Ophthalmology \\ and NIHR Biomedical Research \\ Centre, Department of Clinical \\ Neurosciences, University of \\ Oxford, Oxford, UK; ${ }^{5}$ Department of \\ Ophthalmology, University Hospital \\ of Cologne, Cologne, Germany; \\ ${ }^{6}$ Department of Ophthalmology, \\ University Paris Est Creteil, Centre \\ Hospitalier Intercommunal de \\ Creteil, Creteil, France; ${ }^{7}$ Department \\ of Ophthalmology, Leiden \\ University Medical Center, Leiden, \\ the Netherlands \\ *These authors contributed equally \\ to this work
}

Purpose: To describe the clinical findings and long-term outcome of patients with chronic central serous chorioretinopathy (cCSC).

Materials and methods: This was a retrospective case series in 52 eyes of 36 patients with a follow-up period of at least 1 year. Extensive ophthalmic examination and a validated questionnaire concerning vision-related quality of life (National Eye Institute Visual Function Questionnaire [NEI-VFQ]-39) were analyzed.

Results: Mean visual acuity showed a significant decline over time of 0.16 logarithm of minimum angle of resolution ([logMAR] range: -0.22 to $1.3 ; P=0.009)$ after a mean follow-up period of 10.6 years. Also, patients reported lower vision-related quality of life based on the NEI-VFQ-39 for almost all categories compared to healthy controls. Macular atrophy was diagnosed more often on optical coherence tomography compared to other diagnostic entities. Retinal pigment epithelium detachments in the macula were documented on optical coherence tomography in $56 \%$ of the patients. A significant thinning of foveal thickness was measured over time compared to unaffected fellow eyes $(P=0.002)$. On long-term follow-up, 13 eyes $(37 \%)$ showed an increase in number of hot spots on fluorescein angiography.

Conclusion: This study indicates that $\mathrm{cCSC}$ is a progressive disease in many patients, causing a progressive decline in visual acuity, accompanied by lower reported vision-related quality of life. In deciding whether or not to treat, the progressive nature of cCSC should be taken into account in this relatively young and often still professionally active patient group.

Keywords: chronic central serous chorioretinopathy, micropulse laser, NEI-VFQ-39, PDT, vision-related quality of life

\section{Introduction}

Central serous chorioretinopathy (CSC) is characterized by fluid accumulation between the neuroretina and retinal pigment epithelium (RPE). ${ }^{1,2}$ It often affects the macula, leading to central vision loss, scotoma, metamorphopsia, and/or micropsia. ${ }^{1,2}$ The accumulation of subretinal fluid (SRF) results from leakage through a dysfunctional RPE with a disrupted outer blood-retina barrier. Dilation, congestion, and hyperpermeability of the choriocapillaris and larger choroidal vessels appear to play a pivotal role in the pathogenesis of CSC. ${ }^{3-5}$ The etiology of CSC is unknown. Risk factors for CSC include the use of corticosteroids, Cushing's disease, pregnancy, and male sex. ${ }^{2,6}$ Recent studies have also found genetic associations. ${ }^{7,8}$

There are two main subtypes of CSC. Acute CSC is characterized by sudden vision loss, due to fluid leakage through a focal pinpoint leak in the RPE, often in association with a small RPE detachment. ${ }^{1,2}$ In most acute CSC patients, the SRF resolves spontaneously within 2-3 months. ${ }^{1}$ Visual acuity (VA) in these patients generally returns to (near-) normal levels. ${ }^{1}$ In the other main subtype, chronic CSC (cCSC), patients have more widespread atrophic RPE abnormalities, as well as more 
extensive choroidal changes on fluorescein angiography (FA) and indocyanine green angiography (ICGA) ${ }^{1,2}$ In contrast to acute CSC, most patients with cCSC have more pronounced central vision loss, and often do not show a spontaneous resolution of SRF. ${ }^{2}$

Although cCSC is one of the most common forms of macular degeneration, ${ }^{1}$ relatively little is known about the long-term visual consequences in these patients. The aim of this study was to evaluate the clinical outcome of patients with cCSC and assess their vision-related quality of life to provide a more accurate prognosis to patients.

\section{Materials and methods}

\section{Participants}

Patients with cCSC and a follow-up of at least 1 year were included in this retrospective observational study. Subjects were recruited from the Department of Ophthalmology of Radboud University Medical Center (Nijmegen, the Netherlands) and the Oxford Eye Hospital (Oxford, UK). All patients gave written informed consent for the use of their data for this retrospective study. The diagnosis of cCSC was defined as the presence of SRF longer than 3 months in at least one eye on optical coherence tomography (OCT), RPE window defects on FA with at least 1 "hot spot", defined as indistinct hyperfluorescent area of leakage and/or diffuse leakage in the affected eye(s), and corresponding hyperfluorescent zones on ICGA when available. Patients with evidence of other retinal diagnoses at the first visit were excluded. This study adhered to the tenets of the Declaration of Helsinki, and approval for the study in Oxford was obtained from the Integrated Research Authority, Essex 2 Research Ethics Committee. The ethical approval for the study in Nijmegen was waived by Commissie Mensgebonden Onderzoek (CMO) of the Radboud University Medical Center in Nijmegen, as all clinical data was obtained retrospectively and the questionnaire was found to be non-invasive and not personally sensitive for the study objects.

The following parameters, when available, were collected from the medical records: VA, disease activity, duration of visual symptoms, (prior) use of corticosteroids, number of treatments, color fundus photography, OCT, FA, and ICGA. Only visits for which VA and information about disease activity, defined as subfoveal fluid on OCT or (when OCT was not available) as stated by the ophthalmologist in the medical records, were included. Additionally, patients were asked to complete a validated questionnaire (Visual Function Questionnaire [VFQ]-39). ${ }^{9}$ For all Dutch patients, a validated Dutch translation of this questionnaire was used. ${ }^{10}$

\section{Clinical evaluation}

Submacular RPE detachments, subfoveal SRF, change in submacular SRF accumulation, and retinal atrophy were scored on OCT. The number of hot spots, defined as indistinct hyperfluorescent areas of leakage, seen on FA and ICGA were registered. Evidence of RPE atrophy was assessed on FA and color fundus photography.

Central foveal thickness (CFT), defined as the distance between the outer part of the internal limiting membrane and the outer part of the external limiting membrane at the central fovea, of the first and the last available OCT scans was measured by two independent graders. Only patients who had follow-up imaging with spectral domain OCT (Spectralis ${ }^{\mathrm{TM}}$; Heidelberg Engineering, Heidelberg, Germany) were included in CFT measurements. The mean of the measurements of both graders was used for further analysis after no significant difference between the graders was confirmed by Student's independent $t$-test $(P>0.05)$. In selected patients, unaffected fellow eyes, defined as eyes in which no central lesions suspect for cCSC were present, were included. The difference in CFT over time was compared between the affected and unaffected eyes using Student's independent $t$-test.

\section{Visual acuity}

The VA of all first visits was compared to the VA of all last known visits using an independent-sample $t$-test. For the VA of the last visit, only patients with inactive disease, defined as absence of SRF on OCT, were included. Additionally, a Pearson's correlation test was performed to determine if the number of episodes of active disease or the number of treatments affected the overall change in VA when comparing the first visit to the last visit.

\section{Vision-related quality of life}

The scores of every individual subcategory of the VFQ-39 were compared to the reference group, which was used for assessment in the original validation of the VFQ-39, ${ }^{9,21}$ using the independent-sample $t$-test. The original validation of the VFQ-39 analyzed the data of the reference group as being normally distributed; therefore, this study also chose this approach. A Pearson's correlation test was performed to analyze if the VA at the end of follow-up was associated with VFQ-39 score. $P<0.05$ was considered statistically significant for all tests.

\section{Results \\ Demographics}

Demographic information is presented in Table 1. The mean number of episodes of active disease per eye was 1.7 (range: 0-6). Each episode was defined as a presence 
Table I Demographics of the study population

\begin{tabular}{llll}
\hline Parameter & Nijmegen & Oxford & Total \\
\hline Patients (affected eyes) & $22(33)$ & $14(19)$ & $36(52)$ \\
Sex (male/female) & $13 / 9$ & $8 / 6$ & $2 \mathrm{I} / 15$ \\
Mean age (years) & 56.9 (range: $42-72)$ & 56.3 (range: 39-8I) & 56.7 (range: 39-8I) \\
Bilateral disease (patients) & $1 \mathrm{I}(50 \%)$ & $5(36 \%)$ & $16(44 \%)$ \\
Reported steroid use (patients) & $6(27 \%)$ & $4(29 \%)$ & $10(28 \%)$ \\
Mean follow-up (years) & 9.8 (range: I-24) & 3.8 (range: I-II) & 7.4 (range: I-24) \\
Mean number of treatments per eye & 3.2 (range: 0-8) & 0.1 (range: 0-I) & 2.1 (range: 0-8) \\
\hline
\end{tabular}

Note: Patients were recruited from the Department of Ophthalmology of Radboud University Medical Center (Nijmegen, the Netherlands) and the Oxford Eye Hospital (Oxford, UK).

of documented SRF followed by a period of OCT-proven absence of SRF.

\section{Clinical course}

When the VA of the first and last visits was compared, excluding the 21 eyes $(40 \%)$ with persistent subfoveal SRF at the final visit, a mean decline of 0.16 logarithm of minimum angle of resolution (logMAR; range: -0.22 to $1.3, P=0.009$ ) was found after a mean follow-up period of 10.6 (range: $1.5-24$ ) years. In the 21 eyes (40\%) with persistent subfoveal SRF at the final visit, a mean decline of $0.22 \log$ MAR (range: -0.35 to $0.66, P=0.003$ ) with a mean follow-up period of 4.5 (range: $1-11)$ years was seen. In 11 of these 21 eyes (52\%), the SRF was continuously present during follow-up. Neither the number of episodes of active disease nor number of treatments had a significant effect on overall change in VA.

\section{Optical coherence tomography}

An overview of OCT findings is displayed in Table 2 and Figure $1 \mathrm{~A}-\mathrm{C}$. In the 13 eyes with a decrease in SRF, eight $(62 \%)$ had received treatment. The seven eyes with an increase of SRF had not received treatment. In the eyes showing fluctuating SRF accumulation, 18 (82\%) had received treatment. The following treatments were performed: 41 micropulse therapy, 23 photodynamic therapy (PDT), one argon-laser treatment, one anecortave acetate treatment, and nine anti-VEGF treatments.

In 14 eyes (24\%), SRF was continuously present on OCT until the last follow-up. In this group, this was also the case in five eyes $(36 \%)$ despite receiving treatment (micropulse therapy [52\%], PDT [29\%], anti-VEGF [14\%], and argonlaser treatment [5\%]).

Posterior cystoid retinal degeneration, described previously by Piccolino et al, was seen in seven eyes of four patients ( $8 \%$; Figure 1D-G). ${ }^{11}$ In three of these seven eyes, a lesion suspected to be choroidal neovascularization developed during follow-up. In three of seven eyes, the posterior cystoid retinal degeneration disappeared completely during follow-up, in two eyes this resolution occurred spontaneously, and in one eye it occurred after PDT (Figure 1H and I). The onset of posterior cystoid retinal degeneration was 2,10 , and 16 years after the reported start of the cCSC in three of the four patients in whom this was recorded (Figure 1D-G).

In 21 eyes (41\%) with SRF and six eyes without SRF, spectral domain OCT was available for evaluation of CFT on follow-up. CFT showed a mean decrease of $15.1 \mu \mathrm{m}$

Table 2 Overview of findings on optical coherence tomography and fluorescein angiography

\begin{tabular}{ll}
\hline Optical coherence tomography & Fluorescein angiography \\
\hline Number of RPE detachments & Number of hot spots at first visit $(\mathrm{n}=45)$ \\
$0=23$ eyes $(44 \%)$ & $\mathrm{I}=\mathrm{I}$ eyes $(29 \%)$ \\
$\geq I=29$ eyes $(56 \%)$ & $2=7$ eyes $(16 \%)$ \\
Subfoveal SRF & $\geq 3=3$ eyes $(7 \%)$ \\
Yes $=40$ eyes $(77 \%)$ & Diffuse leakage $=7$ eyes $(16 \%)$ \\
No $=12$ eyes $(23 \%)$ & No clear leakage $=15$ eyes $(33 \%)$ \\
Outer-photoreceptor-layer atrophy & Change in number of hot spots $(\mathrm{n}=35)$ \\
Yes $=24$ eyes $(49 \%)$ & Increase $=13$ eyes $(37 \%)$ \\
No $=28$ eyes $(51 \%)$ & Decrease $=9$ eyes $(26 \%)$ \\
Change in height of SRF accumulation $(n=49)$ & No change $=2$ eyes $(6 \%)$ \\
Decrease $=13(27 \%)$ & New hot spot ${ }^{\mathrm{a}, \mathrm{b}}=2$ eyes $(6 \%)$ \\
Increase $=7(14 \%)$ & No clear hot spot ${ }^{\mathrm{c}}=9$ eyes $(26 \%)$ \\
No change $=3(6 \%)$ &
\end{tabular}

Notes: aHot spot defined as indistinct hyperfluorescent area of leakage; bdisappearance of the original hot spot and appearance of a new hot spot; ${ }^{c}$ no distinct hot spot at first visit. Abbreviations: RPE, retinal pigment epithelium; SRF, subretinal fluid. 

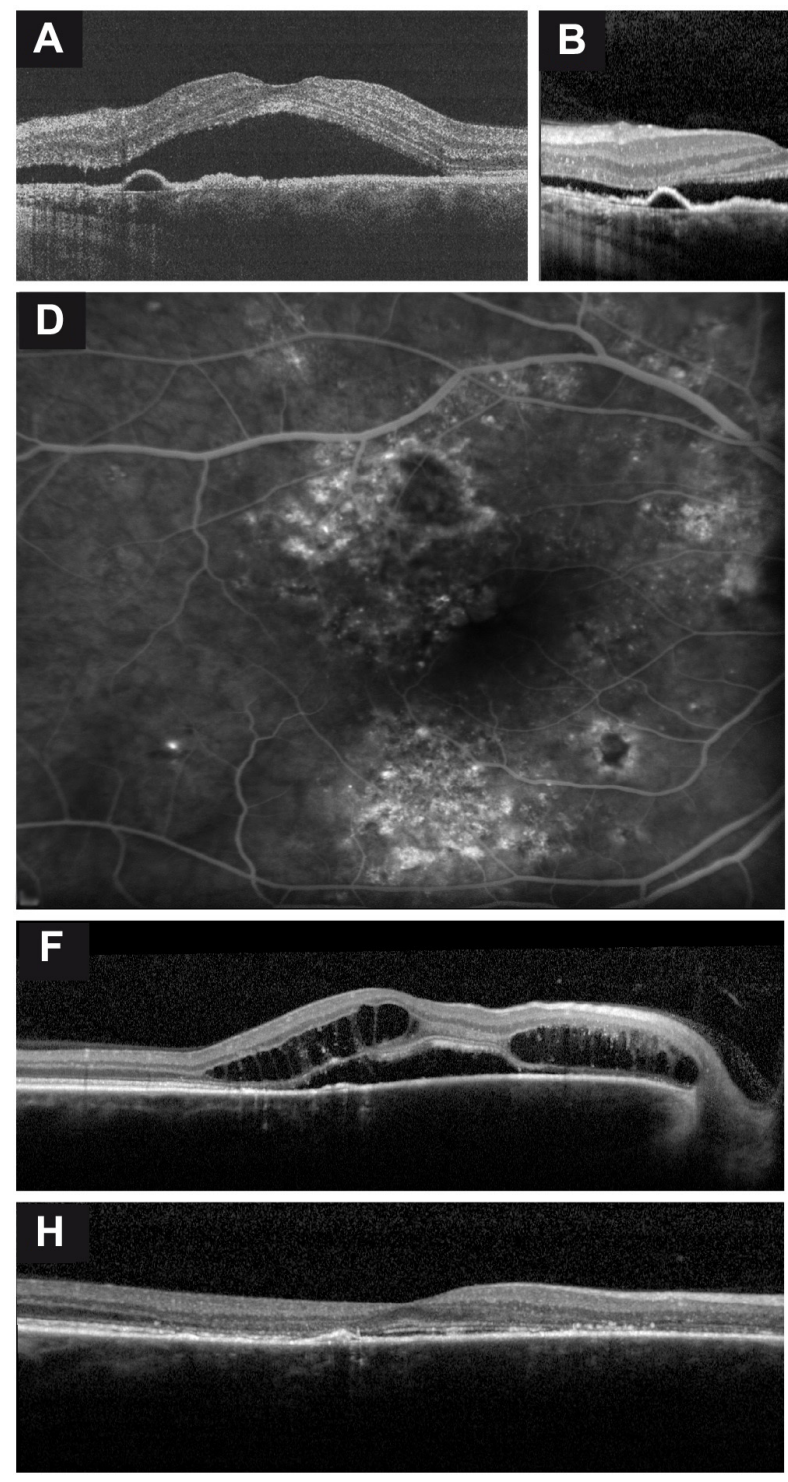
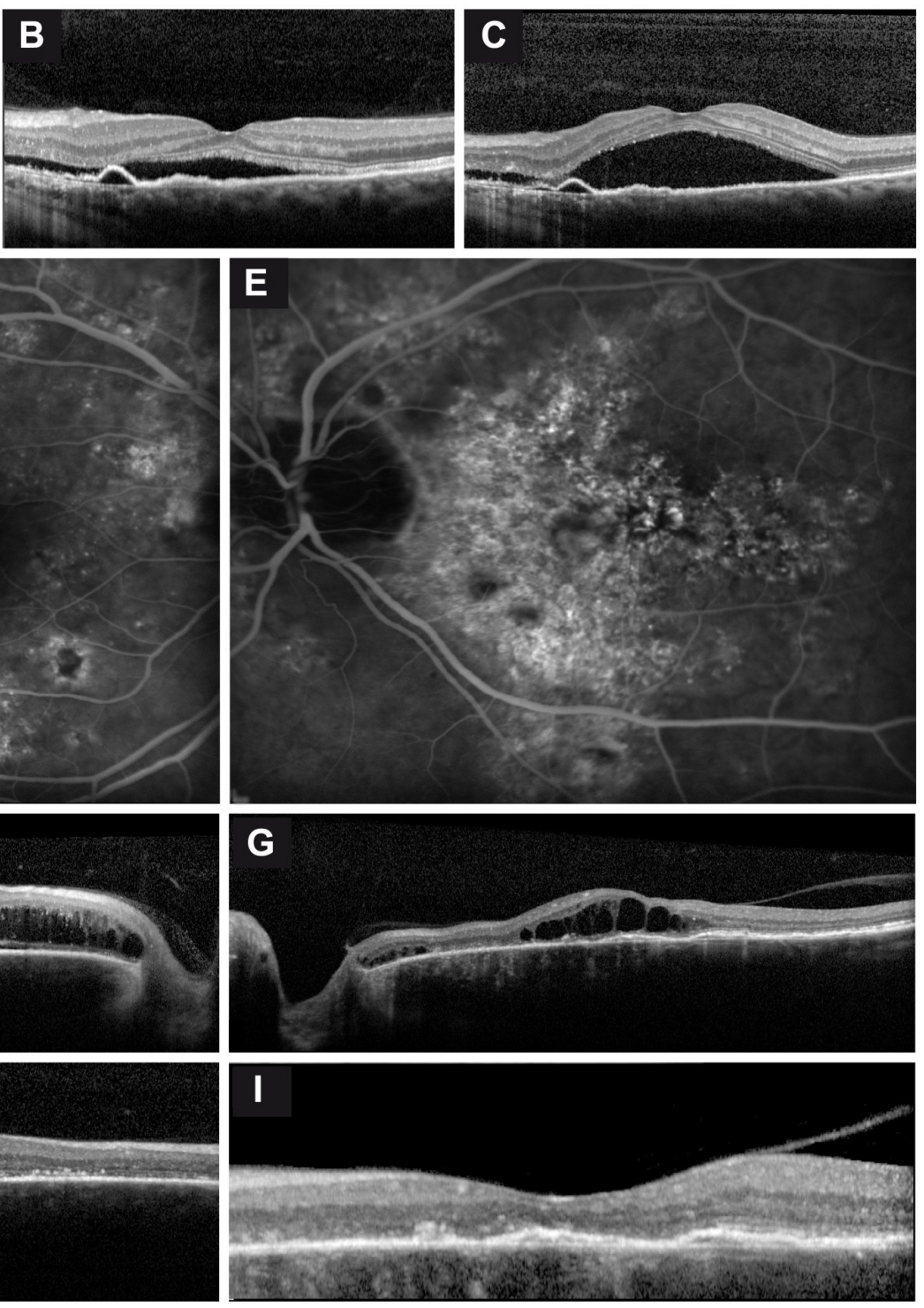

Figure I Imaging of two chronic central serous chorioretinopathy (cCSC) patients.

Notes: Images demonstrate characteristic findings in long-standing cCSC on optical coherence tomography (OCT) and fluorescein angiography (FA). (A-C) Fluctuating subretinal fluid (SRF) accumulation on OCT in the right eye of a patient, and typical subfoveal retinal pigment epithelium detachments. The time between scans $\mathbf{A}$ and $\mathbf{B}$ was I month, in which a clear decrease in SRF occurred, and between scans B and C another 2 weeks elapsed, showing a spontaneous increase in SRF. No therapeutic interventions had been performed between these visits. (D-G) FA and OCT of the right and left eyes of a cCSC patient suffering from bilateral extensive cCSC. On FA, a large area of hyperfluorescence can be seen, indicating advanced disease (D, E). OCT shows not only serous SRF in the right eye but also bilateral central posterior cystoid degeneration, indicative of long-standing disease (F, G). This central posterior cystoid degeneration resolved spontaneously after a period of approximately 6 months $(\mathbf{H}, \mathbf{I})$.

(standard deviation: $17.7 \mu \mathrm{m}$ ) in eyes with SRF, whereas no significant change was seen in eyes without SRF. This difference in CFT was statistically significant $(P=0.002)$.

\section{Fluorescein angiography, indocyanine green angiography, and color fundus photography}

Characteristics of hot spots of leakage on FA on follow-up are shown in Table 2. Of the nine eyes that showed a decrease in number of hot spots during follow-up, in seven the original hot spots disappeared after treatment, whereas the number of hot spots decreased spontaneously in the remaining two eyes. Overall, the mean number of hot spots at the first visit was 1 (range: 0-4) compared to 1.7 (range: 0-7) at the last visit (Figure 2A and B). An increase in atrophic RPE changes on FA was seen in 14 eyes (41\%) during a mean follow-up of 9.7 years (range: 2-24 years). A classic "gravitational tract" was seen in eight eyes $(15 \%)$, and was already present at the first visit in five eyes (Figure 2C and D). The other three eyes developed the gravitational tract after approximately 2,4 , and 8 years, respectively.

When ICGA was available (in 28 eyes [54\%]), hot spots and hyperfluorescent areas on ICGA in 27 eyes (96\%), was 

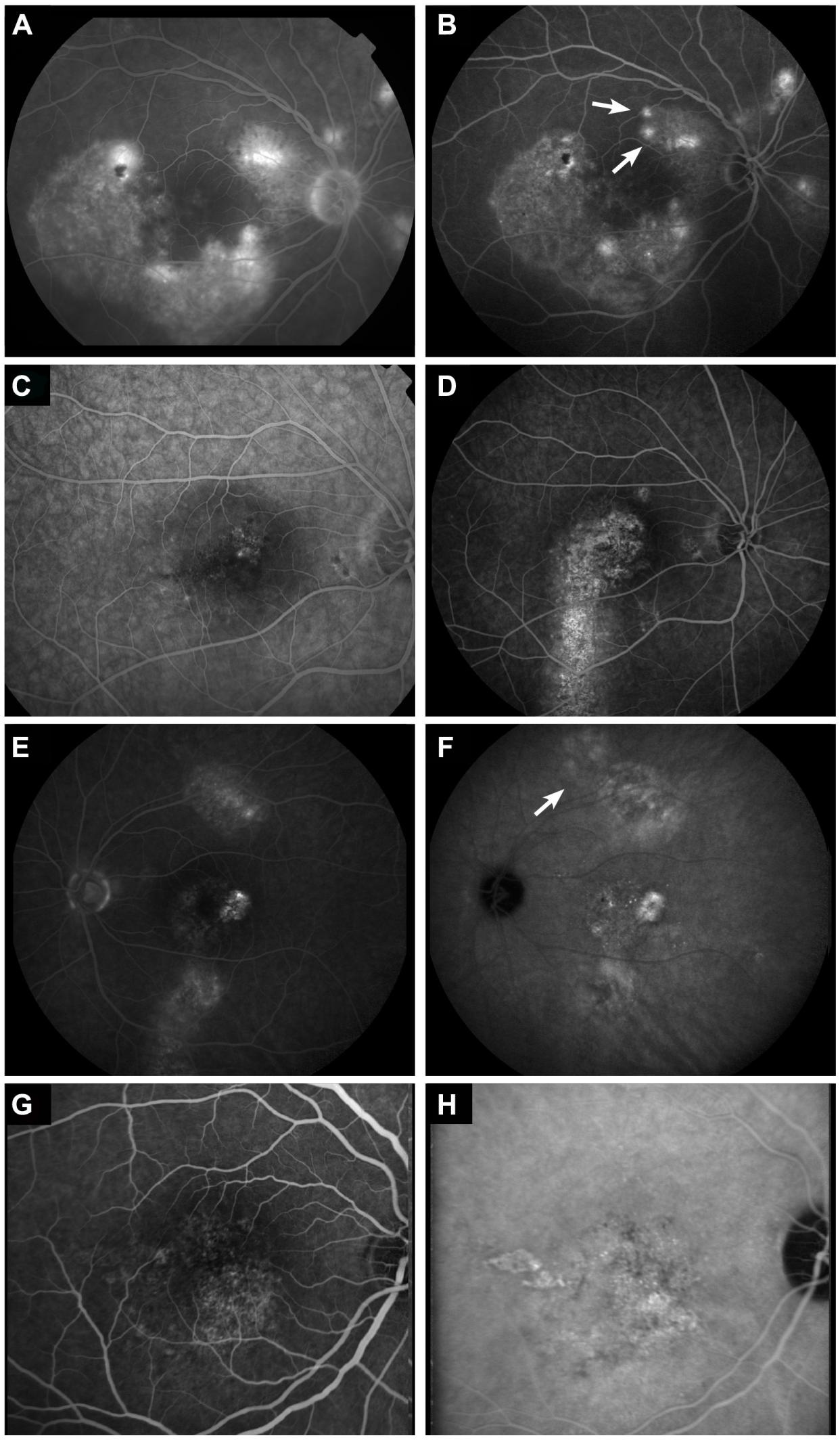

Figure 2 Abnormalities on fluorescein angiography (FA), indocyanine green angiography (ICGA), and fundus autofluorescence in chronic central serous chorioretinopathy. Notes: (A, B) The appearance of "new" hot spots, highlighted by two arrows, on FA during 4 years of follow-up. (C, D) The development of a gravitational tract was observed originating from the initial leakage area after 6 years of follow-up. (E, F) Examples of differences seen in the extent of the hyperfluorescence areas between $F A$ (E) and ICGA (F). The area depicted by the arrow shows clear hyperfluorescence on ICGA, but is not evident on the mid-phase FA. (G, H) ICGA (H) shows small punctiform hyperfluorescent areas in addition to diffuse hyperfluorescence. The corresponding FA (G) of the same patient shows an area of hyperfluorescence that is significantly smaller than the area seen on ICGA. 
Table 3 VFQ-39 questionnaire scores of the study population and controls, and Pearson's correlation with last visual acuity

\begin{tabular}{|c|c|c|c|c|c|c|c|c|}
\hline \multirow[t]{2}{*}{ VFQ-39 item } & \multicolumn{3}{|c|}{ Chronic CSC patients } & \multicolumn{3}{|l|}{ Controls } & \multicolumn{2}{|c|}{$\begin{array}{l}\text { Pearson's correlation } \\
\text { Last visual acuity }\end{array}$} \\
\hline & Subjects & Mean & SD & Subjects & Mean & SD & $P$-value & $P$-value \\
\hline General health & 36 & 65 & 17 & 122 & 75 & 17 & -0.146 & 0.396 \\
\hline General vision & 36 & 62 & 18 & 122 & 81 & 13 & -0.36 & 0.031 \\
\hline Ocular pain & 36 & 85 & 17 & 122 & 90 & 15 & -0.044 & 0.8 \\
\hline Near activities & 36 & 72 & 23 & 122 & 93 & 10 & -0.435 & 0.008 \\
\hline Distance activities & 36 & 79 & 20 & 122 & 95 & 8 & -0.475 & 0.003 \\
\hline Social functioning & 36 & 90 & 17 & 122 & 99 & 3 & -0.416 & 0.012 \\
\hline Mental health & 36 & 72 & 22 & 122 & 91 & 11 & -0.509 & 0.002 \\
\hline Role difficulties & 36 & 74 & 22 & 122 & 96 & 6 & -0.628 & $<0.001$ \\
\hline Dependence & 36 & 91 & 18 & 122 & 99 & 5 & -0.408 & 0.013 \\
\hline Driving & 31 & 70 & 29 & 122 & 89 & 14 & -0.584 & 0.001 \\
\hline Color vision & 36 & 94 & 19 & 122 & 98 & 8 & -0.109 & 0.527 \\
\hline Peripheral vision & 36 & 80 & 26 & 122 & 97 & 10 & -0.178 & 0.299 \\
\hline
\end{tabular}

Abbreviations: CSC, central serous chorioretinopathy; SD, standard deviation; VFQ, Visual Function Questionnaire.

compared with characteristics on FA that was performed on the same date. The overall hyperfluorescent area was larger on ICGA in eight eyes $(30 \%)$, in eleven eyes $(41 \%)$ the hyperfluorescent area had comparable size, in one eye (4\%) the hyperfluorescent area was smaller on ICGA, and in eight eyes (30\%) no clear hyperfluorescence was seen on ICGA to be compared (Figure 2E and F). In eleven eyes (39\%), ICGA showed multiple small punctate hyperfluorescent spots, without clear evidence of a leaking hot spot focus (Figure $2 \mathrm{G}$ and $\mathrm{H}$ ).

Color fundus photography was available at baseline in 42 eyes (81\%), and mild RPE atrophy in the macula was seen in 18 eyes (43\%). Follow-up images were available for 31 eyes (74\%), with a mean follow-up of 45 months (range: 1.5-87 months), and evidence of development and/or progression of RPE atrophy was present in 14 eyes (45\%).

\section{Quality of life}

Patients of this cCSC cohort scored significantly less on the VFQ-39 questionnaire compared to the reference group on eight of the 12 subcategories (Table 3 ). Patients with a lower last recorded VA scored significantly lower on the following categories of the VFQ-39: general vision, near activities, distance activities, social functioning, mental health, role difficulties, dependence, and driving (Table 3).

\section{Discussion}

This study shows that $\mathrm{cCSC}$ is a progressive chorioretinopathy with a significant impact on VA and vision-related quality of life. Patients in this study cohort generally showed a progressive decline in VA, as well as a gradual decrease in CFT on OCT. Wang et al demonstrated in the pre-OCT era that persistence of SRF for more than 4 months can result in foveal atrophy. ${ }^{12}$ This permanent damage to photoreceptors and the RPE can explain the often-persistent visual complaints, even after resolution of SRF. The progressive nature of cCSC was demonstrated on FA, characterized by an increase of hyperfluorescent atrophic RPE changes and an increased number of hot spots during follow-up.

An interesting finding in this study were the small punctate hyperfluorescent spots on ICGA (Figure 2H). Similar lesions were described by Tsujikawa et al. ${ }^{13}$ This group described small clusters, which were located within the center of the focal hyperfluorescent area on ICGA and thus thought to be very small leakage spots. In this study, no clear abnormalities corresponding to these lesions on ICGA could be identified on OCT. However, Lehmann et al identified similar hyperfluorescent lesions on ICGA that correlated with vascular dilations on en face enhanced-depth OCT. ${ }^{14}$ In contrast to the lesions seen in this study, the lesions were always located under an area of SRF accumulation and/or RPE detachment. Additionally, a clinical and pathogenetic overlap between CSC and polypoidal choroidal vasculopathy may exist. Punctate hyperfluorescent spots on ICGA have also been identified in polypoidal choroidal vasculopathy, in which these lesions correlated with a thicker choroid. ${ }^{15} \mathrm{We}$ thus hypothesize that these lesions may be focal vascular dilations in the choriocapillaris that fill on ICGA.

The blood flow in the choroid is among the highest in the human body, and the macular choriocapillaris is thicker, has a distinct lobular architecture, and higher blood flow compared to the peripheral choroid. ${ }^{16,17}$ These anatomical and functional choroidal differences, as well as anatomical differences between central and peripheral RPE and Bruch's 
membrane, ${ }^{18,19}$ probably explain why CSC principally affects the posterior pole. However, it is currently unclear why some individuals develop active CSC in the case of a dysfunctional thickened choroid ("pachychoroid") unlike others, ${ }^{20}$ and why in the same individual the disease can remain subclinical in the fellow eye despite the presence of similar choroidal abnormalities. ${ }^{5}$ It is likely that additional factors play a role in determining an individual's likelihood to develop CSC, for instance, patient-specific differences in genetic background, ${ }^{7,8}$ local differences in RPE outer blood-retina function, and possibly the interphotoreceptor matrix. ${ }^{21}$

This studies data showed a marked decrease in reported vision-related quality of life in this cCSC cohort when compared to healthy individuals. Nevertheless, the impact of cCSC on vision-related quality of life seems to be less pronounced than in other common maculopathies, such as neovascular age-related macula degeneration and diabetic retinopathy. ${ }^{22,23}$ In this cCSC cohort, social functioning and dependence seemed to be most affected. Differences between the vision-related quality of life impact of the different diseases may not only be due to the variable effects on macular anatomy and visual function but may also be partly explained by the difference in the mean age of the different study populations during which the VFQ-39 questionnaire was taken (present study 55 years, neovascular age-related macular degeneration 77 years, diabetic retinopathy 68 years, and diabetic macular edema 62 years). ${ }^{23}$ Also, mean years since diagnosis was far shorter in the neovascular age-related macular degeneration studies compared to the present study (0.6 and 7.4 years). A possible explanation could also be that younger individuals are more flexible and capable of coping with newly developed visual impairment than older individuals. ${ }^{24}$ Also, the negative impact on reported visionrelated quality of life appears to be higher when evaluated closer to the onset of visual impairment.

Surprisingly, a significant difference in the reported quality of color vision was not found, although impaired color vision has been previously reported in CSC. ${ }^{25}$ It is still possible that disturbed color vision exists in this cohort, but that it remains subclinical, for example, due to a better-seeing fellow eye, and does not interfere with daily activities.

\section{Conclusion}

cCSC is a progressive chorioretinopathy, with many cCSC patients experiencing significant vision loss, lower visionrelated quality of life, and lower rating for overall health compared to healthy individuals. Despite these observations, it is not uncommon for ophthalmologists to adopt a conservative approach in these patients, who are often relatively young. The findings of this study demonstrate that the possible impact of cCSC should not be underestimated, and thus an active treatment approach may be advocated. However, few high-quality randomized controlled treatment trials have been reported in cCSC, with a current lack of a gold standard for treatment of cCSC. We are currently performing a multicenter prospective randomized controlled treatment trial, comparing half-dose PDT with high-density subthreshold micropulse-laser treatment in cCSC (EudraCT 2012-004555-36, NCT01797861) in an attempt to identify the most suitable treatment strategy in cCSC.

\section{Acknowledgments}

The authors thank Joannes MM Groenewoud (PhD) for his statistical support and input to this manuscript. This study was supported by the Macula Vision Research Foundation, MD Fonds, Landelijke Stichting voor Blinden en Slechtzienden, Gelderse Blindenstichting, Stichting Nederlands Oogheelkundig Onderzoek, Stichting Blindenhulp, Stichting AF Deutman Oogheelkunde Researchfonds, Nijmeegse Oogonderzoek Stichting, Janivo Stichting, and Oogfonds. The sponsor or funding organization had no role in the design or conduct of this research. Dr Camiel JF Boon is now employed by the Department of Ophthalmology, Acedemic Medical Center Amsterdam.

\section{Disclosure}

Dr Camiel JF Boon was supported by a Niels Stensen Fellowship. The authors report no conflicts of interest in this work.

\section{References}

1. Liew G, Quin G, Gillies M, Fraser-Bell S. Central serous chorioretinopathy: a review of epidemiology and pathophysiology. Clin Exp Ophthalmol. 2013;41(2):201-214.

2. Nicholson B, Noble J, Forooghian F, Meyerle C. Central serous chorioretinopathy: update on pathophysiology and treatment. Surv Ophthalmol. 2013;58(2):103-126.

3. Guyer DR, Yannuzzi LA, Slakter JS, Sorenson JA, Ho A, Orlock D. Digital indocyanine green videoangiography of central serous chorioretinopathy. Arch Ophthalmol. 1994;112(8):1057-1062.

4. Prünte C, Flammer J. Choroidal capillary and venous congestion in central serous chorioretinopathy. Am J Ophthalmol. 1996;121(1):26-34.

5. Kim YT, Kang SW, Bai KH. Choroidal thickness in both eyes of patients with unilaterally active central serous chorioretinopathy. Eye (Lond). 2011;25(12):1635-1640.

6. Bouzas EA, Karadimas P, Pournaras CJ. Central serous chorioretinopathy and glucocorticoids. Surv Ophthalmol. 2002;47(5):431-448.

7. Miki A, Kondo N, Yanagisawa S, Bessho H, Honda S, Negi A. Common variants in the complement factor $\mathrm{H}$ gene confer genetic susceptibility to central serous chorioretinopathy. Ophthalmology. 2014;121(5): 1067-1072.

8. de Jong EK, Breukink MB, Schellevis RL, et al. Chronic central serous chorioretinopathy is associated with genetic variants implicated in agerelated macular degeneration. Ophthalmology. 2015;122(3):562-570. 
9. Mangione CM, Lee PP, Gutierrez PR, Spritzer K, Berry S, Hays RD. Development of the 25-item National Eye Institute Visual Function Questionnaire. Arch Ophthalmol. 2001;119(7):1050-1058.

10. Van der Sterre G, Van der Graaf E, Verezen C, et al. Visual Function Questionnaire - 25: National Eye Institute Nederlandse Consensus Vertaling versie 2001 (VFQ-25/NL). 2013. Available from: http:// www.erasmusmc.nl/mage/publicaties/aanvullingen/3503529. Accessed October 9, 2016.

11. Piccolino FC, De La Longrais RR, Manea M, Cicinelli S. Posterior cystoid retinal degeneration in central serous chorioretinopathy. Retina. 2008;28(7):1008-1012.

12. Wang MS, Sander B, Larsen M. Retinal atrophy in idiopathic central serous chorioretinopathy. Am J Ophthalmol. 2002;133(6):787-793.

13. Tsujikawa A, Ojima Y, Yamashiro K, et al. Punctate hyperfluorescent spots associated with central serous chorioretinopathy as seen on indocyanine green angiography. Retina. 2010;30(5):801-809.

14. Lehmann M, Wolff B, Vasseur V, et al. Retinal and choroidal changes observed with 'En face' enhanced-depth imaging OCT in central serous chorioretinopathy. Br J Ophthalmol. 2013;97(9):1181-1186.

15. Park SJ, Kim BH, Park KH, Woo SJ. Punctate hyperfluorescence spot as a common choroidopathy of central serous chorioretinopathy and polypoidal choroidal vasculopathy. Am J Ophthalmol. 2014;158(6): 1155-1163.

16. Nickla DL, Wallman J. The multifunctional choroid. Prog Retin Eye Res. 2010;29(2):144-168.

17. Alm A, Bill A. Ocular and optic nerve blood flow at normal and increased intraocular pressures in monkeys (Macaca irus): a study with radioactively labelled microspheres including flow determinations in brain and some other tissues. Exp Eye Res. 1973;15(1):15-29.
18. Boulton M, Dayhaw-Barker P. The role of the retinal pigment epithelium: topographical variation and ageing changes. Eye (Lond). 2001; 15(Pt 3):384-389.

19. Ramrattan RS, van der Schaft TL, Mooy CM, de Bruijn WC, Mulder PG, de Jong PT. Morphometric analysis of Bruch's membrane, the choriocapillaris, and the choroid in aging. Invest Ophthalmol Vis Sci. 1994; 35(6):2857-2864.

20. Warrow DJ, Hoang QV, Freund KB. Pachychoroid pigment epitheliopathy. Retina. 2013;33(8):1659-1672.

21. Hageman GS, Marmor MF, Yao XY, Johnsen LV. The interphotoreceptor matrix mediates primate retinal adhesion. Arch Ophthalmol. 1995; 113(5):655-660.

22. Suner IJ, Kokame GT, Yu E, Ward J, Dolan C, Bressler NM. Responsiveness of NEI VFQ-25 to changes in visual acuity in neovascular AMD: validation studies from two phase 3 clinical trials. Invest Ophthalmol Vis Sci. 2009;50(8):3629-3635.

23. Lloyd AJ, Loftus J, Turner M, Lai G, Pleil A. Psychometric validation of the Visual Function Questionnaire-25 in patients with diabetic macular edema. Health Qual Life Outcomes. 2013;11:10.

24. Cheng C, Lau HP, Chan MP. Coping flexibility and psychological adjustment to stressful life changes. A meta-analytic review. Psychol Bull. 2014;140(6):1582-1607.

25. Maaranen TH, Tuppurainen KT, Mäntyjärvi MI. Color vision defects after central serous chorioretinopathy. Retina. 2000;20(6):633-637.
Clinical Ophthalmology

\section{Publish your work in this journal}

Clinical Ophthalmology is an international, peer-reviewed journal covering all subspecialties within ophthalmology. Key topics include: Optometry; Visual science; Pharmacology and drug therapy in eye diseases; Basic Sciences; Primary and Secondary eye care; Patient Safety and Quality of Care Improvements. This journal is indexed on Submit your manuscript here: http://www.dovepress.com/clinical-ophthalmology-journal

\section{Dovepress}

PubMed Central and CAS, and is the official journal of The Society of Clinical Ophthalmology (SCO). The manuscript management system is completely online and includes a very quick and fair peer-review system, which is all easy to use. Visit http://www.dovepress.com/ testimonials.php to read real quotes from published authors. 